\title{
ANÁLISE DA DISPENSAÇÃO DE ANTIDEPRESSIVOS E ANSIOLITTICOS EM UMA FARMÁCIA COMERCIAL DO NOROESTE DO RIO GRANDE DO SUL
}

\author{
Analysis of antidepressants and anxiolytics dispensation in a commercial \\ pharmacy in the northwest of Rio Grande Do Sul state
}

\author{
Carine Agostini Zuanazzi ${ }^{1}$; Neiva Aparecida Grazziotin ${ }^{2}$
}

${ }^{1}$ Acadêmica do Curso de Farmácia da Universidade Regional Integrada do Alto Uruguai e das Missões - URI Erechim.E-mail: carine.zuanazzi@live.com

${ }^{2}$ Farmacêutica Bioquímica, Mestre em Ciências Biológicas, Docente do Departamento de Ciências da Saúde da Universidade Regional Integrada do Alto Uruguai e das Missões - URI Erechim.

Data do recebimento: 29/07/2019 - Data do aceite: 19/02/2020

RESUMO: Considerando o crescente aumento de pessoas com depressão e ansiedade, devido a condições estressantes que afetam a vida cotidiana, além de o uso prolongado dos psicotrópicos causar dependência química, o objetivo deste estudo foi analisar a dispensação de medicamentos antidepressivos e ansiolíticos em uma farmácia comercial localizada na cidade de Paim Filho, RS. Trata-se de um estudo transversal retrospectivo. A amostra foi composta pelos medicamentos antidepressivos e ansiolíticos dispensados na farmácia, referente ao período de janeiro a dezembro de 2018. A coleta de dados teve início após a aprovação do estudo pelo Comitê de Ética em Pesquisa da URI Erechim, com a transcrição desses medicamentos e quantidade dispensada, disponíveis em um banco de dados na farmácia. As informações foram transcritas para um banco de dados elaborado no programa Excel. Foram utilizadas medidas descritivas. Os resultados mostraram que, dentre os antidepressivos dispensados pela farmácia, a classe terapêutica prevalente foi a dos Inibidores Seletivos da Recaptação de Serotonina (ISRS) e, dentre os ansiolíticos, a classe terapêutica predominante foi a dos benzodiazepínicos. Quando avaliados os medicamentos antidepressivos, destaca-se a paroxetina. E, em relação aos medicamentos ansiolíticos, o mais utilizado foi o alprazolam.

Palavras-chave: Antidepressivos. Ansiolíticos. Inibidores Seletivos da Recaptação de Serotonina. Benzodiazepínicos. 


\begin{abstract}
Considering the increasing number of people with depression and anxiety due to stressful conditions that affect daily life, in addition to the prolonged use of psychotropic drugs, the objective of this study was to analyze the dispensing of antidepressant and anxiolytic drugs in a commercial pharmacy located in the city of Paim Filho / RS. It is a cross-sectional retrospective study. The sample consisted of the antidepressant and anxiolytic drugs dispensed at the Pharmacy from January to December 2018. Data collection began after the approval of the study by the Research Ethics Committee of URI Erechim, with the transcription of these drugs and quantity dispensed, available in a database at the pharmacy. The information was transcribed to a database prepared in the Excel program. Descriptive measures were used. The results showed that, among the antidepressants dispensed by the pharmacy, the most prevalent therapeutic class was Selective Serotonin Reuptake Inhibitors (SSRIs), and among the anxiolytics, the predominant therapeutic class was benzodiazepines. When evaluating antidepressant drugs, paroxetine stands out. And, for anxiolytic drugs, alprazolam was the most used.
\end{abstract}

Keywords: Antidepressants. Anxiolytics. Selective Serotonin Reuptake Inhibitors. Benzodiazepines.

\section{Introdução}

O uso de drogas psicotrópicas vem aumentando nos últimos anos, possivelmente por causa das novas exigências do mundo moderno. Os medicamentos são importantes para o tratamento das mais diversas doenças, dentre elas os transtornos mentais, para os quais são utilizados, principalmente, os psicotrópicos (NASÁRIO; SILVA, 2016).

Levando em consideração todas as tribulações do dia a dia, com relação a vida familiar, trabalho, trânsito intenso, luto, traumas psicológicos, entre outros fatores, uma das alternativas que as pessoas procuram é a utilização de medicamentos psicotrópicos para dormir melhor ou para ter maior rendimento no trabalho (NASÁRIO; SILVA, 2016).

Segundo a Organização Mundial da Saúde (OMS), o número estimado de pessoas com depressão, em todo o mundo, em 2015, foi de 300 milhões, sendo as mulheres mais afeta- das. As taxas de prevalência variam de acordo com a idade, ocorrendo principalmente na idade adulta. Acorre, também, em crianças e adolescentes com menos de 15 anos, porém em níveis mais baixos. Em casos extremos, pode levar ao suicídio (WHO, 2017). Estudos realizados no período de 2005 a 2015 mostram um aumento de $18 \%$ na incidência da depressão, considerada a principal causa de incapacidade em todo o mundo (NAÇÕES UNIDAS BRASIL, 2017). Um levantamento realizado pela OMS sobre ansiedade constatou que $9,3 \%$ da população brasileira manifesta este quadro e 5,8\%, transtornos depressivos (WHO, 2017).

Em se tratando de cuidados com a saúde, as mulheres procuram com mais frequência ajuda médica do que os homens. Isso se dá, principalmente, porque o homem, na visão do senso comum, é um ser mais forte que dificilmente adoece, além disso é condicionado desde pequeno a negar seus sentimentos, assim a procura pelos serviços de saúde apre- 
senta predominância feminina (LEVORATO et al., 2014).

Fatores como violência, gravidez, problemas de saúde e a posição na família são indicadores do estado de humor depressivo em mulheres. Entretanto, outros fatores podem estar associados a esse estado de depressão, como, por exemplo, o rompimento de uma relação, a mudança de cidade, estado ou país (CORREIA; BORLOTI, 2011).

Considerando o crescente aumento de pessoas com depressão e ansiedade, devido a condições estressantes que afetam a vida cotidiana, além de que o uso prolongado dos psicotrópicos causa dependência química, $o$ presente trabalho teve como objetivo analisar a dispensação de medicamentos antidepressivos e ansiolíticos em uma farmácia comercial localizada no noroeste do Rio Grande do Sul no período de janeiro a dezembro de 2018.

\section{Material e Métodos}

A pesquisa seguiu um modelo de estudo transversal retrospectivo. A amostra do estudo foi composta pelos medicamentos antidepressivos e ansiolíticos dispensados, no ano de 2018, em uma farmácia localizada na cidade de Paim Filho, RS.

A coleta de dados foi realizada por meio da transcrição dos medicamentos antidepressivos e ansiolíticos dos relatórios disponíveis no banco de dados da farmácia. As variáveis de interesse foram os medicamentos antidepressivos e ansiolíticos, a classe terapêutica e a quantidade dispensada. As informações foram transcritas para um banco de dados elaborado no programa Excel. Foram utilizadas medidas descritivas. Os resultados foram expressos como frequência absoluta (n) e frequência relativa (\%).

A pesquisa foi aprovada pelo Comitê de Ética em Pesquisa da Universidade Regional Integrada do Alto Uruguai e das Mis- sões - URI Erechim, sob o parecer número 3.079.808.

\section{Resultados e Discussão}

Foram analisados os relatórios, disponibilizados por uma farmácia localizada na cidade de Paim Filho, RS, referentes aos medicamentos antidepressivos e ansiolíticos dispensados no ano de 2018. Dentre estes medicamentos, 1.114 caixas $(58,9 \%)$ foram de antidepressivos e 776 caixas $(41,1 \%)$, de ansiolíticos.

Estes dados se confirmam em um estudo que foi realizado na cidade de São Paulo em 2017 sobre o uso de psicotrópicos em adultos e idosos, em que destacaram-se o uso dos antidepressivos (52,6\%) (PRADO; FRANCISCO; BARROS, 2017). No estudo realizado em uma farmácia comunitária no município de Sobral, CE, que avaliou o uso de psicotrópicos, os antidepressivos também foram os mais comercializados, $32,13 \%$ (COSTA; OLIVEIRA, 2017).

A Tabela I mostra que os antidepressivos mais dispensados para os pacientes pertencem à classe dos Inibidores Seletivos da Recaptação de Serotonina (ISRS) com 692 caixas de medicamentos, o que corresponde a $62,11 \%$.

Resultado semelhante foi encontrado em estudo realizado na Estratégia de Saúde da Família de Sobral, Ceará, nos anos de 2010 e 2011, sendo os Inibidores Seletivos da Recaptação de Serotonina a classe mais escolhida pelos médicos. Os antidepressivos foram prevalentes com $34,75 \%$ dos psicotrópicos distribuídos pela Central de Abastecimento Farmacêutico (CAF), seguidos por antiepiléticos $(33,99 \%)$, antipsicóticos, benzodiazepínicos e anticolinérgicos (ARAÚJO et al., 2012). Já em uma revisão sistemática, realizada por Magalhães et al. (2016), o maior padrão de consumo de antidepressivos em di- 
ferentes estados (São Paulo, Santa Catarina e Minas gerais) esteve dividido entre os ISRS e os antidepressivos tricíclicos, de acordo com artigos analisados no período de 2004 a 2013.

Dentre os motivos pelos quais os Inibidores Seletivos da Recaptação de Serotonina são os mais indicados, destaca-se uma maior eficácia terapêutica, menor toxicidade, pouca interferência nos outros sistemas e menor incidência de efeitos colaterais (COUTINHO; NETO FILHO, 2010).

Tabela I - Frequência dos antidepressivos agrupados por classe terapêutica na Farmácia Santa Terezinha, no município de Paim Filho, RS, no ano de 2018

\begin{tabular}{lcc}
\hline Classe Terapêutica & n (caixas) & $\begin{array}{c}\text { Frequência } \\
(\%)\end{array}$ \\
\hline ISRS & 692 & 62,11 \\
Tricíclico & 135 & 12,11 \\
IRSN & 206 & 18,49 \\
Atípico & 67 & 6,01 \\
Tricíclico/ & 14 & 1,25 \\
Benzodiazepínico & & 100 \\
\hline TOTAL & 1114 & \\
\hline
\end{tabular}

ISRS: Inibidores Seletivos da Recaptação de Serotonina, IRSN: Inibidores da Recaptação de Serotonina e Noradrenalina

Observa-se, na Tabela II, que o antidepressivo da classe terapêutica dos Inibidores Seletivos da Recaptação de Serotonina (ISRS) mais prescrito foi a paroxetina, com 292 caixas $(26,20 \%)$, seguida por escitalopram, com 207 caixas $(18,60 \%)$ de um total de 692 caixas pertencentes à classe em questão. Contudo, o medicamento de menor dispensação foi Clomipramina, o qual pertence à classe dos Antidepressivos Tricíclicos.

Os dados encontrados divergem dos de outros estudos. Para Araújo et al. (2012), o antidepressivo de maior prevalência foi a amitriptilina, com 699.187 unidades, o que representou 44,62\% da distribuição da referida classe, seguida da fluoxetina, com 424.593 unidades, correspondente a $27,09 \%$. Já para Ribeiro et al. (2014), em sua pesquisa com estudantes de medicina de uma faculdade pública paulista, o antidepressivo mais citado foi a fluoxetina, sendo que, dentre os 289 alunos entrevistados, 33 utilizavam ou já utilizaram antidepressivos. Um estudo realizado com professores de escolas de diferentes redes de ensino em um município do norte do Rio Grande do Sul revelou que os antidepressivos mais usados foram fluoxetina com 32,43\%, seguido de sertralina com 27,05\% (SEGAT, DIEFENTHAELER, 2013).

A Tabela III revela que, dentre as 776 caixas de ansiolíticos dispensados para os pacientes do presente estudo, as mais prescritas pertencem à classe dos Benzodiazepínicos com 524 caixas de medicamentos, o que corresponde a $67,52 \%$.

Em estudos realizados por Azevedo et al. (2016), Torres et al. (2014) e Marchi et al. (2013), os ansiolíticos mais mencionados também foram os da classe de benzodiazepínicos, isso se dá por serem os medicamentos mais seguros em relação às outras classes.

Apesar dos benzodiazepínicos serem empregados no tratamento de diversas doenças psiquiátricas e não psiquiátricas, e normalmente seguros e tolerados, o potencial para a utilização inadequada e excessiva dos mesmos é considerável (MOTA et al., 2010).

A grande preocupação é devido ao seu potencial para o abuso, dependência, abstinência, tolerância, sedação, prejuízos psicomotores e sua interação com o álcool e com outros medicamentos. É importante salientar que o álcool contribui para as mortes envolvendo o uso de benzodiazepínicos (CRUZ et al., 2006).

A principal aplicação terapêutica dos benzodiazepínicos é como ansiolítico e hipnótico, mas também é utilizado como anti- 
Tabela II - Antidepressivos mais utilizados na Farmácia Santa Terezinha, no município de Paim Filho, RS, no ano de 2018

\begin{tabular}{lccl}
\hline Antidepressivo & n (caixas) & Frequência (\%) & Classe Terapêutica \\
\hline Paroxetina & 292 & 26,20 & ISRS \\
Escitalopram & 207 & 18,60 & ISRS \\
Sertralina & 112 & 10,05 & ISRS \\
Citalopram & 42 & 3,80 & ISRS \\
Fluoxetina & 32 & 2,90 & ISRS \\
Fluvoxamina & 7 & 0,60 & ISRS \\
Amitriptilina & 100 & 9 & Tricíclico \\
Nortriptilina & 20 & 1,80 & Tricíclico \\
Maprotilina & 8 & 0,70 & Tricíclico \\
Imipramina & 5 & 0,45 & Tricíclico \\
Clomipramina & 2 & 0,20 & Tricíclico \\
Duloxetina & 113 & 10,15 & IRSN \\
Venlafaxina & 67 & 6 & IRSN \\
Desvenlafaxina & 26 & 2,3 & IRSN \\
Trazodona & 57 & 5,10 & Atípico \\
Bupropiona & 10 & 0,90 & Atípico \\
Amitriptilina+clordiazepóxido & 14 & 1,25 & Tricíclico/Benzodiazepínico \\
\hline TOTAL & 1114 & 100 & \\
\hline
\end{tabular}

ISRS: Inibidores Seletivos da Recaptação de Serotonina, IRSN: Inibidores da Recaptação de Serotonina e Noradrenalina

convulsivante, relaxante muscular e sedativo. Estão entre os medicamentos mais prescritos no mundo, muitas vezes sem indicação adequada, constituindo um grave problema de saúde pública. Quando bem indicados, são úteis por apresentarem rápido início de ação, poucos efeitos colaterais e boa margem de segurança (CANCELLA, 2012).

A Tabela IV mostra que, dentre os ansiolíticos da classe terapêutica dos benzodiazepínicos, os mais dispensados foram Alprazolam com 216 caixas $(27,85 \%)$, e Clonazepam com 155 caixas (19,98\%), seguido de Fenobarbital com 105 caixas $(13,55 \%)$, pertencente a classe dos barbitúricos.
Tabela III - Frequência dos ansiolíticos agrupados por classe terapêutica na Farmácia Santa Terezinha no município de Paim Filho, RS, no ano de 2018

\begin{tabular}{lcc}
\hline Classe Terapêutica & $\begin{array}{c}\mathbf{n} \\
\text { (caixas) }\end{array}$ & $\begin{array}{c}\text { Frequência } \\
\mathbf{( \% )}\end{array}$ \\
\hline Benzodiazepínico & 524 & 67,52 \\
Barbitúrico & 105 & 13,53 \\
Compostos Z & 70 & 9,02 \\
Outros & 63 & 8,11 \\
Neuroléptico/ & 14 & 1,80 \\
Benzodiazepínico & & 100 \\
\hline TOTAL & 776 & \\
\hline
\end{tabular}


Azevedo et al. (2016) analisaram a distribuição e frequência de consumo de ansiolíticos benzodiazepínicos, verificaram que o Alprazolam se manteve no topo, seguido por Bromazepam, Clonazepam, Lorazepam e Diazepam. A coleta teve base em um banco de dados da ANVISA (Agência Nacional de Vigilância Sanitária) e uma amostra de 27 capitais brasileiras.

Torres et al. (2014) avaliaram 1570 prescrições médicas de psicotrópicos aviadas no ano de 2012 em uma farmácia comercial do município de Santa Inês, MA, sendo que os medicamentos benzodiazepínicos foram os mais prescritos: Clonazepam $(33,82 \%)$, Bromazepam (15,85\%), Diazepam (15,62\%) e Cloxazolam $(12,68 \%)$.

Marchi et al. (2013) avaliaram a incidência de ansiedade e o consumo de ansiolíticos entre estudantes de enfermagem de uma universidade pública de Ribeirão Preto, SP. Os medicamentos mais consumidos pelos estudantes foram Diazepam (37,5\%), Clonazepam (16,7\%), Bromazepam $(8,3 \%)$ e Lorazepam (6,3\%). Participaram do estudo 308 estudantes, e destes $16 \%$ usavam ou já usaram algum tipo de ansiolítico.

A parada do uso destes medicamentos não deve ser feita de forma abrupta, mas sim de maneira gradual, sendo que sua retirada deve ser feita em um período de 6 a 8 semanas (NUNES; BASTOS, 2016).

Há muito tempo, a prescrição de antidepressivos e ansiolíticos deixou de ser exclusividade dos psiquiatras, tornando-se um problema de saúde pública, sendo que várias distorções constatadas nas prescrições de psicotrópicos têm sido praticadas pelas mais diversas especialidades médicas. Geralmente, quem mais prescreve esses medicamentos

Tabela IV - Ansiolíticos mais utilizados na Farmácia Santa Terezinha, no município de Paim Filho, RS, no ano de 2018

\begin{tabular}{lccl}
\hline Ansiolítico & n (caixas) & Frequência (\%) & Classe Terapêutica \\
\hline Alprazolam & 216 & 27,85 & Benzodiazepínico \\
Clonazepam & 155 & 19,98 & Benzodiazepínico \\
Bromazepam & 52 & 6,70 & Benzodiazepínico \\
Lorazepam & 37 & 4,76 & Benzodiazepínico \\
Diazepam & 33 & 4,25 & Benzodiazepínico \\
Clobazam & 23 & 2,96 & Benzodiazepínico \\
Cloxazolam & 4 & 0,50 & Benzodiazepínico \\
Flunitrazepam & 2 & 0,25 & Benzodiazepínico \\
Flurazepam & 2 & 0,25 & Benzodiazepínico \\
Fenobarbital & 105 & 13,55 & Barbitúrico \\
Zolpidem & 70 & 9,05 & Compostos Z \\
Buspirona & 63 & 8,10 & Outros \\
Sulpirida+bromazepam & 14 & 1,80 & Neuroléptico/Benzodiazepínico \\
\hline TOTAL & 776 & 100 & \\
\hline
\end{tabular}


são os médicos clínicos gerais, uma vez que os usuários procuram os medicamentos primeiramente nas Unidades Básicas de Saúde (UBS). Um clínico geral não preparado tem mais dificuldade em diagnosticar um transtorno mental, levando a um consumo indiscriminado de medicamentos psicotrópicos, especialmente antidepressivos e ansiolíticos (COSTA; OLIVEIRA, 2017). Desta maneira, as diferenças de medicamentos antidepressivos e ansiolíticos identificadas neste trabalho, em relação a outros estudos, podem estar relacionadas à especialidade do médico prescritor, além de diferenças regionais, ou também pelo fato de determinado medicamento ter um custo menor que o outro, tornando mais acessível o tratamento ao paciente e, consequentemente, favorecendo a adesão ao tratamento.

\section{Considerações Finais}

A classe de antidepressivos predominante foi a dos Inibidores Seletivos da Recaptação de Serotonina (ISRS) com $62,11 \%$, o que pode estar relacionado ao fato de serem medicamentos que apresentam menos efeitos adversos em relação às demais classes.
Quanto aos antidepressivos mais prescritos destacam-se Paroxetina, Escitalopram, Duloxetina e Sertralina.

Com relação aos ansiolíticos, a classe prevalente foi a dos benzodiazepínicos com $67,52 \%$, possivelmente por serem mais seguros e apresentarem menor potencial de intoxicação quando comparados às outras classes. Dentre os ansiolíticos mais prescritos podemos destacar Alprazolam, Clonazepam, Fenobarbital e Zolpidem.

Ressalta-se a importância de que a indicação tanto de antidepressivos quanto dos ansiolíticos seja realizada de forma criteriosa. Além disso, é necessário que os pacientes sejam orientados acerca do uso racional destas medicações, evitando seu consumo excessivo ou sem orientação médica.

Enfim, reforça-se a necessidade de um acompanhamento médico aos usuários destes medicamentos desde o início até o fim do tratamento e, também, na retirada dos mesmos. Destaca-se a importância de um farmacêutico para orientações e esclarecimento de dúvidas com relação aos medicamentos, efeitos adversos, interações farmacológicas e modo de usar.

\section{REFERÊNCIAS}

ARAÚJO, L. L. et al. Distribuição de antidepressivos e benzodiazepínicos na estratégia de saúde da família de Sobral-CE. SANARE - Revista de Políticas Públicas, v. 11, n. 1, p. 45-54, 2012.

AZEVEDO, A. J.; ARAUUJO, A. A.; FERREIRA, M. A. Consumo de ansiolíticos benzodiazepínicos: uma correlação entre dados do SNGPC e indicadores sociodemográficos nas capitais brasileiras.

Revista Ciência \& Saúde Coletiva, v. 21, n. 1 p. 83-90, 2016.

CANCELLA, D. Análise do uso de psicofármacos na Atenção Básica Primária: uma revisão da literatura. UFMG - Curso de Especialização em Atenção Básica em Saúde da Família. Belo Horizonte, 2012.

CORREIA, M. K.; BORLOTI, E. Mulher e Depressão: Uma Análise Comportamental-Contextual.

Revista Acta Comportamentalia, v. 19, n. 3, p. 359-373, 2011. 
COSTA, G. M.; OLIVEIRA, M. A. Estudos das prescrições de psicotrópicos em uma farmácia da cidade de Sobral, Ceará, Brasil. Infarma - Ciências Farmacêuticas, v. 29, n. 1, p. 27-33, 2017.

COUTINHO, P. K.; NETO FILHO, M. A. Depressão: Conceito e tratamento. Uningá Review, v. 4, n. 3, p. 50-55, 2010.

CRUZ, A. V. et al. Uso crônico de diazepam em idosos atendidos na rede pública em Tatuí-SP.

Revista Ciência Farmacêutica Básica e Aplicada, v. 27, n. 3, p. 259-267, 2006.

LEVORATO et al. Fatores associados à procura por serviços de saúde numa perspectiva relacional de gênero. Revista Ciência \& saúde coletiva, v. 19, n. 4, p. 1263-1274, 2014.

MAGALHÃES et al. Psicotrópicos: perfil de prescrições de benzodiazepínicos, antidepressivos e anorexígenos a partir de uma revisão sistemática. Eletronic Journal of Pharmacy, v. 13, n. 3, p. 111-122, 2016.

MARCHI, K. C. et al. Ansiedade e consumo de ansiolíticos entre estudantes de enfermagem de uma universidade pública. Revista Eletrônica de Enfermagem, v. 15, n. 3, p. 731-739, 2013.

MOTA, D. M. et al. Uso abusivo de benzidamina no Brasil: uma abordagem em farmacovigilância. Ciência Saúde Coletiva, v. 15, n. 3, p. 717-724 2010.

NAÇÕES UNIDAS DO BRASIL. Depressão afeta mais de 300 milhões de pessoas e é doença que mais incapacita pacientes. 2017. Disponível em: https://nacoesunidas.org/depressao-afetamais-de-300-milhoes-de-pessoas-e-e-doenca-que-mais-incapacita-pacientes-diz-oms. Acesso em: 01 set. 2018.

NASARIO, M.; SILVA, M. M. O consumo excessivo de medicamentos psicotrópicos na atualidade. In: Pós-graduação de saúde mental e atenção psicossocial (lato sensu) no centro universitário para o desenvolvimento do Alto Vale do Itajaí - UNIDAVI. [2016?]. Disponível em: www.uniedu. sed.sc.gov.br/wp-content/uploads/2016/02/Marcela-Nasario.pdf. Acesso em: 24 set. 2018.

NUNES, B. S.; BASTOS, F. M. Efeitos colaterais atribuídos ao uso indevido e prolongado de benzodiazepínicos. Saúde e Ciência em Ação, v. 3, n. 1, p. 71-82, 2016.

PRADO, M.; FRANSCISCO, P.; BARROS, M. Uso de medicamentos psicotrópicos em adultos e idosos residentes em Campinas, São Paulo: um estudo transversal de base populacional. In: Universidade Estadual de Campinas, Faculdade de Ciências Médicas. São Paulo, 2017.

RIBEIRO, A. G. et al. Antidepressivos: uso, adesão e conhecimento entre estudantes de medicina. Revista Ciência \& Saúde Coletiva, v. 19, n. 6, p. 1825-1833, 2014.

SEGAT, E.; DIEFENTHAELER, H. S. Uso de medicamentos antidepressivos por professores de escolas de diferentes redes de ensino em um município do norte do Rio Grande do Sul. Revista Perspectiva, v. 37, n. 137, p. 45-54, 2013.

TORRES, M. L. et al. Prescrição de psicotrópicos e especialidade médica: estudo em uma farmácia comercial no município de Maranhão. Revista Científica do ITPAC, v. 7, n. 4, Pub. 4, 2014.

WHO. World Health Organization. Depression and other common mental disorders: global health estimates. Geneva: World Health Organization, 2017. Disponível em: https://www.who.int/mental health/management/depression/prevalence_global_health_estimates/en/. Acesso em: 02 mar. 2020. 